\title{
MRA Image Segmentation with Capillary Active Contour
}

\author{
Pingkun Yan and Ashraf A. Kassim \\ Department of Electrical \& Computer Engineering, National University of Singapore \\ \{pingkun, ashraf\}@nus.edu.sg
}

\begin{abstract}
Precise segmentation of three-dimensional (3D) magnetic resonance angiography (MRA) image can be a very useful computer aided diagnosis (CAD) tool in clinical routines. Our objective is to develop a specific segmentation scheme for accurately extracting vasculature from MRA images. Our proposed algorithm, called the capillary active contour (CAC), models capillary action where liquid can climb along the boundaries of thin tubes. The CAC, which is implemented based on level sets, is able to segment thin vessels and has been applied for verification on synthetic volumetric images and real 3D MRA images. Compared with other state-of-the-art MRA segmentation algorithms, our experiments show that the introduced capillary force can facilitate more accurate segmentation of blood vessels.
\end{abstract}

\section{Introduction}

Magnetic resonance angiography (MRA) is a noninvasive medical imaging modality that produces three-dimensional (3D) images of vessels and cavities. Accurate extraction of 3D vascular structures from MRA images has become increasingly important for diagnosis and quantification of vascular diseases.

Existing MRA segmentation techniques can be broadly divided into two categories: skeleton-based and nonskeleton-based. Skeleton-based techniques are those indirect methods which segment and reconstruct the vessels by first detecting the centerlines of the vessels. Several methods have been developed based on this principle and multiscale schemes to allow for the diversity of vessel sizes [1, 2, 3]. In these approaches, the centerline models can be generated explicitly, implicitly or via postprocessing by vessel modeling methods. Contrary to above methods, nonskeleton-based techniques are those that compute the vessels in 3D directly. In this category, deformable model based methods have received considerable attention and success.

Klein et al. 4 proposed to reconstruct 2D vessel boundaries or 3D vessel walls using deformable surface models represented by B-spline surfaces. However, it is not possible to employ parameterized deformable models to effectively deal with whole vessel trees, as the models would be required to change topology during evolution. Yim et al. [5] proposed a deformable surface model based on triangulated meshes for vessel construction in 3D. Nevertheless, it may be problematic to apply these methods [4,5] for segmentation of vessels from low contrast MRA 


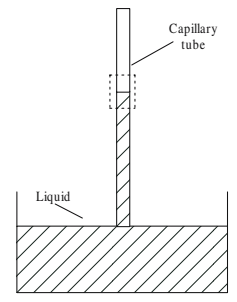

(a)

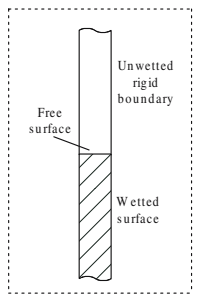

(b)

Fig. 1. Capillary action. (a) Capillary tube, (b) Surfaces of a three-phase system.

images. Geometric deformable models based on level set theory [6,7, which can freely adapt into complex topologies of objects, were then proposed and applied on MRA images segmentation. Chen and Amini 8 employed a hybrid model using both parametric and geometric deformable models for segmentation of an entire vascular tree. However, these methods may have difficulty in extracting tiny vessels from 3D images. Small vessels and their branches, which exhibit much variability, are very important in planning and performing neurosurgical procedures. Greater details can provide more precise navigation and localization information for computer guided procedures. Lorigo et al. 9] proposed the "CURVES" algorithm to extract thin vessels, which uses geodesic active contour 7 based on the co-dimension two level set method [10]. However, to make the evolving curve stop at the object boundaries, the evolution speed needs to be multiplied by a heuristic factor.

Our work aims to develop an image segmentation methodology for automatically extracting the whole vasculature from 3D angiography. Inspired by the capillary action associated with thin tubes as shown in Fig. 1(a), in which liquid climbs up to some height without external pulling force, an algorithm for vasculature extraction is proposed. In the capillary action, the thinner the tube, the higher the liquid level in the tube. The situation is very similar to the segmentation of blood vessels if we imagine thin blood vessels as capillary tubes. Hence, it may be useful to employ this mechanism for segmenting thin vessels in low contrast situations. By modeling this phenomenon and fitting it into image segmentation problem, the capillary active contour (CAC) is obtained for MRA image segmentation.

\section{Capillary Geodesic Active Contour}

The capillary action can be understood as an energy minimization process, which involves surface tensions. We first introduce the energy functional associated with the free surface and wetted surface. Following that, the volume constraint of the fluid is considered. To minimize the overall energy functional, the EulerLagrange equations are computed for each part and these derived parts are 
integrated together to get the final evolution equation of the fluid surface. Since the capillary force is incorporated, the derived method is called capillary active contour.

\subsection{Free Surface Energy}

Free surface is the part of the liquid surface that is not in contact with the solid boundaries. Capillary action can only be observed when the adhesion tension between molecules of fluid and molecules of solid is larger than the cohesion tension between fluid molecules. Therefore, molecules on the free surface tend to be attracted toward the solid boundaries. Fluid molecules will have lower potential energy when they are nearer to solid boundaries and this energy will be minimized when they are on the solid boundaries.

The gradient magnitude $|\nabla I|$ is calculated to indicate possible boundaries, where $I:[0, a] \times[0, b] \times[0, c] \rightarrow \mathbb{R}^{+}$denotes an image. A uniform decreasing function $g:\left[0,+\infty\left[\rightarrow \mathbb{R}^{+}\right.\right.$is defined, where $g(r) \rightarrow 0$ as $r \rightarrow+\infty$. Function $g(|\nabla I(S(q))|)$ is used to describe the energy coefficient associated with the surface $S(q):[0,1] \rightarrow \mathbb{R}^{3}$, which is the surface tension coefficient in physics. Let $S_{f}(t)$ denote the free surface of the liquid at time $t$. The capillary energy of free surface can be expressed as

$$
E\left(S_{f}(t)\right)=\int_{q} g(S(q))\left|\frac{\partial S(t, q)}{\partial q}\right| d q
$$

by integrating over $q$, where $S(t, q) \in S_{f}(t)$. By computing the Euler-Lagrange of (11), the evolution equation of the free surface is obtained as

$$
\boldsymbol{S}_{t}=g \kappa \mathcal{N}-(\nabla g \cdot \mathcal{N}) \mathcal{N}
$$

where $\kappa$ is the Euclidean curvature, and $\mathcal{N}$ is the unit normal vector of the surface.

\subsection{Wetting Surface Energy}

Since the adhesion force is larger than the cohesion force, the fluid molecules will be attracted to the solid boundaries and the unwetted surface is then converted into wetted surface. The total energy involved in this dynamic process can be modeled as

$$
E\left(S_{w}(t)\right)=\beta S_{w}+\beta^{*} S_{w}^{*},
$$

where $S_{w}$ is the area wetted by the fluid and $S_{w}^{*}$ is the area in contact with the outer medium (unwetted surface). Since $S_{w}$ can be extended in an arbitrarily continuous way into $S_{w}^{*}$, we have

$$
E\left(S_{w}(t)\right)=\hat{\beta} S_{w}^{*}
$$

where $\hat{\beta}=\beta^{*}-\beta$. Thus, minimizing the wetted surface energy is equivalent to minimizing the surface $S_{w}^{*}$ through evolving the contact line. However, solving 

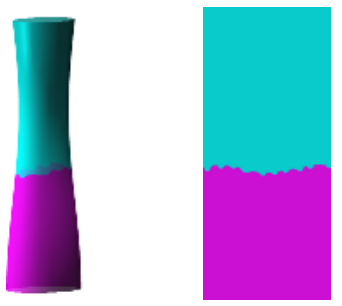

Fig. 2. 3D tubular surface is stretched to get $2 \mathrm{D}$ surface

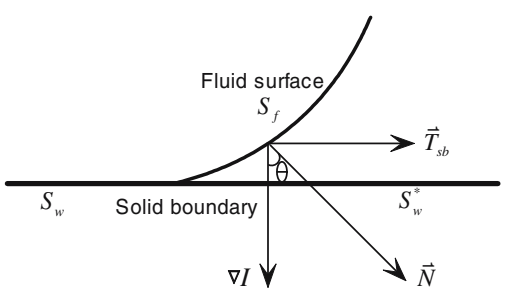

Fig. 3. The evolving direction of the contact line

this problem directly in $3 \mathrm{D}$ is problematic, because the surface to be minimized is not enclosed by the contact line. Since we are only interested in an immediate neighborhood of the contact line, it is always possible to find some view point that all the surface area is under the contact line (see Fig. 2). In addition, the $3 \mathrm{D}$ surface can be "cut" along a line and then stretched to becoming a 2D plane as shown in Fig. 2,

Let $\mathcal{C}(t, x):[0,1] \rightarrow \mathbb{R}^{2}$ denote the contact line between the fluid surface and the unwetted surface. The length of the curve $\int\left|\frac{\partial \mathcal{C}(t, x)}{\partial x}\right| d x$ is considered as a regularization term to keep the curve smooth. Thus, the energy associated with the wetted surface can be written as

$$
E\left(S_{w}(t)\right)=\int \mathcal{C}(t, x) d x+\lambda \int\left|\frac{\partial \mathcal{C}(t, x)}{\partial x}\right| d x .
$$

where $\lambda$ is a real positive constant parameter. Then the $2 \mathrm{D}$ evolving equation of contact line $\mathcal{C}$ is obtained as

$$
\mathcal{C}_{t}=(1+\lambda \hat{\kappa}) \mathcal{N}_{\Gamma},
$$

where $\hat{\kappa}$ and $\mathcal{N}_{\Gamma}$ are the Euclidean curvature and the unit normal vector of the contact line in $2 \mathrm{D}$, respectively. However, when working in $3 \mathrm{D}$, the curvature $\hat{\kappa}$ of the 2D contact line becomes a co-dimension two curvature $\hat{\kappa}_{2}$ 10, 9 . Furthermore, as we are considering the evolution of liquid along vessel walls, the direction of evolution should be parallel to the wall at each point, i.e., the tangential direction $\mathcal{T}_{s b}$ as shown in Fig. 3. Then (6) is changed into

$$
\mathcal{C}_{t}=\left(1+\lambda \hat{\kappa}_{2}\right) \mathcal{T}_{s b}
$$

The tangential subvector can be calculated as

$$
\mathcal{T}_{s b}=\mathcal{N}-\frac{\nabla g}{|\nabla g|} \cos \theta,
$$

where $\cos \theta=\mathcal{N} \cdot \frac{\nabla g}{|\nabla g|}$. 
Thus, the overall evolution equation describing the energy of wetting surfaces can be written as

$$
\boldsymbol{S}_{t}=\left(1+\lambda \hat{\kappa}_{2}\right)\left(\mathcal{N}-\frac{\nabla g}{|\nabla g|} \cos \theta\right) .
$$

\subsection{Volume Constraint}

The volume constraint is considered as $E(V)=c V$, which can be expanded as

$$
E(V)=c \int S(t, q) d q,
$$

where $c$ is a Lagrange multiplier. By applying the gradient descent minimization, we have evolution equation $\boldsymbol{S}_{t}=\boldsymbol{c} \mathcal{N}$, which is a constant velocity for minimizing the volume enclosed by the surface. Since liquid is bounded by solid, the fluid surface will snap to the boundaries. Thus, the constant velocity is multiplied by function $g$ and then the evolution equation is modified into

$$
\boldsymbol{S}_{t}=g(S(q)) \boldsymbol{c} \mathcal{N}
$$

\subsection{Evolution Equation}

Based on the results obtained separately in the above sections, the final evolution equation is obtained by integrating these terms

$$
\boldsymbol{S}_{t}=g(\kappa+c) \mathcal{N}-(\nabla g \cdot \mathcal{N}) \mathcal{N}+\alpha\left(1+\lambda \hat{\kappa}_{2}\right)\left(\mathcal{N}-\frac{\nabla g}{|\nabla g|} \cos \theta\right),
$$

where parameter $\alpha$ is a real positive constant. The constant term $c$ in (11) acts like balloon force in [11, which facilitates the evolving surface snapping to solid boundaries. Comparing the new speed function (11) with those of the geodesic active contour [7], the third term is new which comes from the capillary action. The new term makes the fluid surface move along the solid boundaries. The capillary action term is expected to facilitate the evolving surface adapting into thin parts of objects, e.g. thin vessels.

\subsection{Level Set Evolution Equation}

According to the level set theory and noting the fact that

$$
\mathcal{N}=-\frac{\nabla \Psi}{|\nabla \Psi|}
$$

evolving a surface $S$ under the speed function (11) is equivalent to updating a volumetric map $\Psi$ with

$$
\Psi_{t}=g(\kappa+c)|\nabla \Psi|+\nabla g \cdot \nabla \Psi+\alpha\left(1+\lambda \hat{\kappa}_{2}\right)|\nabla \Psi|\left(1-\cos ^{2} \theta\right)
$$

where $S$ is the zero level set embedded in $\Psi$ and

$$
\cos \theta=\frac{\nabla \Psi \cdot \nabla g}{|\nabla \Psi||\nabla g|}
$$




\section{Results and Discussion}

\subsection{Capillary Force}

Fig. 4 shows the application of the $\mathrm{CAC}$ in a computer generated cylinder, which illustrates the effects of the capillary force. Starting from the initialization visualized in Fig. 4(b), evolution is done according to the level set speed function in (12). In our experiment, all the parameters are fixed except that $\alpha$ is varying. The evolution results with different parameter settings after 50 iterations are visualized in Fig. 4 .

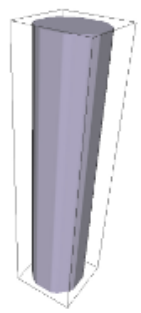

(a)

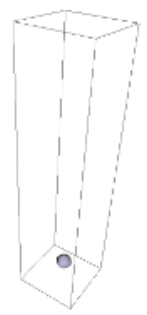

(b)

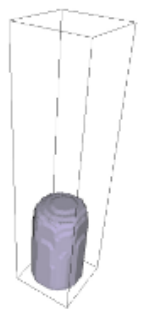

(c)

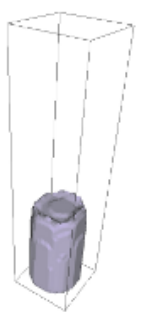

(d)

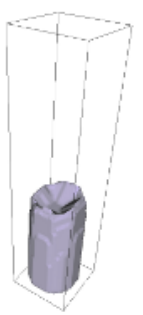

(e)

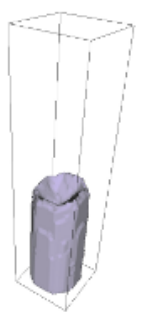

(f)

Fig. 4. Illustration of the effects on varying capillary force coefficient $\alpha$. (a) Generated cylinder. (b) Initialization of the algorithm. (c) $\alpha=0$. (d) $\alpha=0.25$. (e) $\alpha=0.5$. (f) $\alpha=0.75$.

When $\alpha$ is set to zero, the CAC in fact evolves exactly like a geodesic active contour 7 . The evolution of the free surface in the axial direction of the tubular object is driven by the mean curvature $\kappa$ and constant speed $c$ in (12). Since this term is multiplied by the uniquely decreasing function $g$, its contribution will be small when the surface is near the object boundary, and becomes zero when the surface is on the edges. Hence, we can see the free surface is convex as shown in Fig. 4(c). When the tubular object becomes much thinner, the evolution may stop because of the small value of $g$ at everywhere. After incorporating the capillary force, the free surface is attracted by the unwetted surface when near the solid boundaries and meniscus [12] can be observed as shown in Fig. [4(d)(e)(f). With larger value of $\alpha$, the surface evolution along the object boundary will be stronger and faster. Therefore, the capillary force can facilitate the evolution of the free surface even when the vessels are very thin.

\subsection{MRA Segmentation}

The CAC method is also applied on 3D MRA images of cerebral vasculature. The image size is $512 \times 512 \times 120$ voxels with spacing $0.43 \mathrm{~mm} \times 0.43 \mathrm{~mm} \times 1.2 \mathrm{~mm}$. The initial surface is obtained by thresholding the raw dataset. The MIP of MRA data set used in our experiments is shown in Fig. 5, To make the results 


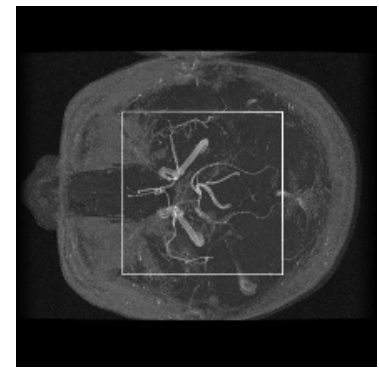

(a) MIP

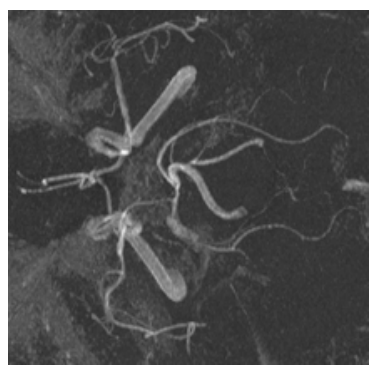

(b) Region of interest

Fig. 5. MIP of the cerebral MRA data set

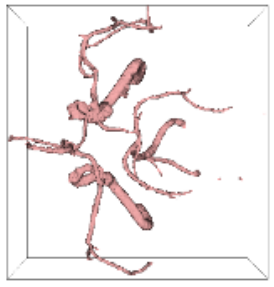

(a)

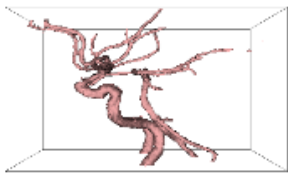

(b)

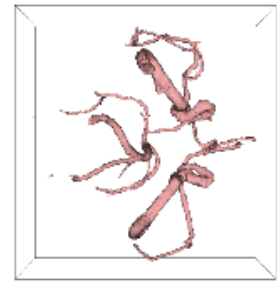

(c)

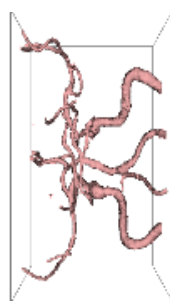

(d)

Fig. 6. MRA segmentation results using CURVES

clearer, a region of interest (ROI) is extracted (Fig. 5(b)) and only vessels inside the ROI are segmented and visualized.

We have compared our proposed CAC method with the state-of-the-art CURVES 9. Identical parameter settings in the evolution equations are used for both methods except for the capillary force coefficient, which is specific to the CAC. The segmentation results of CURVES and CAC are shown in Fig. 6

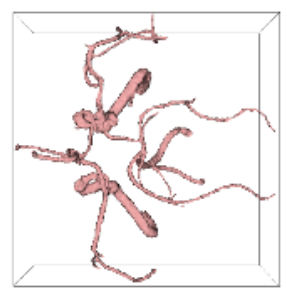

(a)

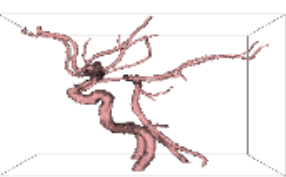

(b)

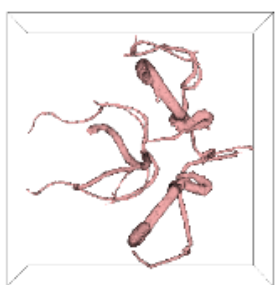

(c)

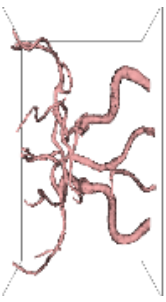

(d)

Fig. 7. MRA segmentation results using CAC 
and Fig. 7 with different points of view. The CURVES algorithm successfully extracts much of the vasculature but fails to identify some parts when compared with the CAC.

\section{Conclusions}

In this paper, we propose the capillary active contour and apply it to both 3D synthetic and cerebral MRA images segmentation. The incorporated capillary force adapts the evolving surface into very thin branches of blood vessels and obtains more accurate segmentation as demonstrated in our experiments. Compared with other techniques, the CAC can achieve more details of vasculature. Our approach is geometric in nature and topology free due to that implicit representation of the evolving surface is used.

\section{References}

1. Frangi, A.F., Niessen, W.J., Hoogeveen, R.M., van Walsum, T., Viergever, M.A.: Model-based quantitation of 3-D magnetic resonance angiographic images. IEEE Trans. Med. Imag. 18 (1999) 946-956

2. Krissian, K., Malandain, G., Ayache, N., Vaillant, R., Trousset, Y.: Model based detection of tubular structures in 3D images. Computer Vision and Image Understanding 80 (2000) 130-171

3. Aylward, S.R., Bullitt, E.: Initialization, noise, singularities, and scale in height ridge traversal for tubular object centerline extraction. IEEE Trans. Med. Imag. 21 (2002) 61-75

4. Klein, A.K., Lee, F., Amini, A.A.: Quantitative coronary angiography with deformable spline models. IEEE Trans. Med. Imag. 16 (1997) 468-482

5. Yim, P.J., Boudewijn, G., Vasbiner, C., Ho, V.B., Choyke, P.L.: Isosurfaces as deformable models for magnetic resonance angiography. IEEE Trans. Med. Imag. 22 (2003) 875-881

6. Malladi, R., Sethian, J.A., Vermuri, B.C.: Shape modeling with front propagation: A level set approach. IEEE Trans. Pattern Anal. and Machine Intell. 17 (1995) $158-174$

7. Caselles, V., Kimmel, R., Sapiro, G.: Geodesic active contours. Int. J. Computer Vision 22 (1997) 61-79

8. Chen, J., Amini, A.A.: Quantifying 3-D vascular structures in MRA images using hybrid PDE and geometric deformable models. IEEE Trans. Med. Imag. 23 (2004) 1251-1262

9. Lorigo, L.M., Faugeras, O., Grimson, W.E.L., Keriven, R., Kikinis, R., Nabavi, A., Westin, C.F.: CURVES: Curve evolution for vessel segmentation. Medical Image Analysis 5 (2001) 195-206

10. Ambrosio, L., Soner, H.M.: Level set approach to mean curvature flow in arbitrary codimension. J. Differential Geometry 43 (1996) 693-737

11. Cohen, L.D.: On active contour models and balloons. CVGIP: Image Understanding 53 (1991) 211-218

12. Finn, R.: Equilibrium Capillary Surfaces. Springer-Verlag, New York (1986) 